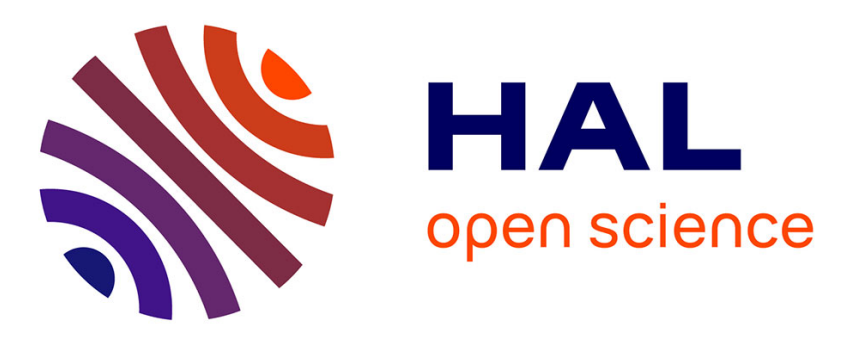

\title{
Target Tracking with Limited Sensing Range in Autonomous Mobile Sensor Networks
}

Jing Bai, Peng Cheng, Jiming Chen, Adrien Guenard, Ye-Qiong Song

\section{To cite this version:}

Jing Bai, Peng Cheng, Jiming Chen, Adrien Guenard, Ye-Qiong Song. Target Tracking with Limited Sensing Range in Autonomous Mobile Sensor Networks. IEEE workshop WiSARN, in conjunction with IEEE 8th International Conference on Distributed Computing in Sensor Systems (DCOSS2012), IEEE, May 2012, Hangzhou, China. pp.329-334. hal-00745238

\section{HAL Id: hal-00745238 \\ https://hal.inria.fr/hal-00745238}

Submitted on 25 Oct 2012

HAL is a multi-disciplinary open access archive for the deposit and dissemination of scientific research documents, whether they are published or not. The documents may come from teaching and research institutions in France or abroad, or from public or private research centers.
L'archive ouverte pluridisciplinaire HAL, est destinée au dépôt et à la diffusion de documents scientifiques de niveau recherche, publiés ou non, émanant des établissements d'enseignement et de recherche français ou étrangers, des laboratoires publics ou privés. 


\title{
Target Tracking with Limited Sensing Range in Autonomous Mobile Sensor Networks
}

\author{
Jing Bai*, Peng Cheng*, Jiming Chen*, Adrien Guenard ${ }^{\dagger}$, Yeqiong Song ${ }^{\dagger}$ \\ * State Key Lab. of Industrial Control Technology, Zhejiang University, Hangzhou, 310027 China \\ ${ }^{\dagger}$ LORIA - University of Lorraine, France \\ Email: jing.bai1987@gmail.com, \{pcheng, jmchen\}@iipc.zju.edu.cn, \{adrien.guenard, song\}@loria.fr
}

\begin{abstract}
As technology advancements in robotics and wireless communication, tracking mobile targets using mobile sensors has aroused widespread concern in recent years. In this paper, we propose a novel coordinative moving strategy for autonomous mobile sensor networks to guarantee the target can be detected in each observed step while minimizing the amount of moving sensors. The proposed scheme consists of obtaining the current position of the target, which is then used to predict the next time-step location of the target. Once the uncertainty region of the target's position is defined, the proposed method allows the mobile sensors to cover it in an optimal way. Therefore, we can assign each mobile sensor to an optimal location to cover the uncertainty region while minimizing the total traveled distance of sensors. Extensive simulations are given to evaluated performance and demonstrate the efficiency of the proposed strategy.

Index Terms-Mobile Sensor Networks; Target Tracking; Coverage; Assignment;
\end{abstract}

\section{INTRODUCTION}

Mobile Sensor Networks (MSN) are composed of a large amount of mobile devices which have computation, communication, sensing and processing abilities. MSN also have a variety of practical applications, such as environment monitoring, security surveillance [1], [2]. Target tracking is a critical problem in the field of sensor networks. Compared with static sensors, tracking in MSN can take advantage of the mobility of the sensors to improve the tracking performance.

Usually, a target can be divided into two classes, i.e., cooperative target and non-cooperative target. The former one can broadcast cooperative signals (e.g., radio frequencies, vibrations, and sound, etc.) from time to time, which keeps on communication with the sensors for detecting the target. The latter one, in a lot of applications, many intelligent targets would not emit such kind of signals. In this case, the sensors have to actively detect the target by frequently broadcasting certain signals, such as infrared or ultrasonic waves. When the target is within the sensing range of the sensors, it can be detected. In this paper, we mainly focus on tracking a noncooperative target.

It is well known that tracking performance largely depends on the dynamic model of target and the measurement model. Commonly, passive infrared (PIR) sensors can detect the

\footnotetext{
${ }^{1}$ The work is partially supported by NSFC under Grants 61004060 60974122, by the Specialized Research Fund for the Doctoral Program of China under Grant 20100101110066. The work is also supported by joint ANR-NSFC Quasimodo project (ANR 2010 INTB 020601 and NSFC 61061130563).
}

changes of infrared radiation energy from the environment, so that these are always used for detecting extern event. Additionally, if there is no strict assumptions on the target dynamic model, ultrasonic sensors can accurately measure the target distance relative to the sensor. Therefore, in this paper, we consider the mobile sensors equipped with both PIR module and ultrasonic sensing module to detect the target's presence and sense the distance between the target and the sensors. In this paper, we focus on making full use of the mobility of the sensors to guarantee that the target will not be lost once being observed. In this case, the mobile sensors dynamically adjust their positions according to the target's motions. So it is of great importance to design a dynamic moving strategy based on the measurements to improve the tracking quality.

It is worth noting that the mobility of the sensors tremendously enhance the performance of the tracking system, but it also brings in certain design challenges. One challenge is that how to choose a minimum set of mobile sensors to move and assigned destinations of each selected sensor. we are able to transform the problem into a coverage problem with mobile sensor networks by utilizing the moving model of target. Since the model itself contains uncertainty, we will focus on the guaranteed capture with minimal number of moving sensors at each step. Moreover, due to the highly constrained energy in MSN, we will further design the moving destinations of sensors at each step so that the total traveled distance would also be minimized.

The rest of the paper is organized as follows. We present a brief review of related literature in Section II. Section III provides the formulation of the target tracking problem. The detailed design and algorithms are depicted in Section IV. The simulation results are shown in Section V, including the results conducted with Matlab as well as Samovar. Finally, Section VI concludes the paper.

\section{RELATED WORKS}

Target tracking is one of the most important applications of wireless sensor networks, such as healthcare, building and military monitoring, home security [3], [4], [5]. So a lot of useful application can benefit greatly from accurate tracking.

Considering the problem of single target tracking in controlled mobility sensor networks, researchers in [6] have 
proposed a novel strategy which is based on the intervalbased estimation for managing sensors mobility to improve the estimation of the position of the target. Mobile sensors are able to move to a new position at each step, which is chosen within the computed set using ant colony optimization algorithm. The movement decision is made upon whether the new position will improve the estimation while minimizing the energy consumption or not. The proposed approach uses a hybrid sensor network. While mobile sensors are not only used for optimizing the performance of the target tracking but also maximizing the lifetime of the network, static sensors guarantee the whole coverage of the network.

The target tracking problem under insufficient anchor coverage is first summarized in [7], which aims to propose a target tracking framework for insufficient anchor coverage and asynchronous networks so that improve the tracking accuracy. Due to lack of costly anchors and environment constraints, the target can not be detected by more than three anchors simultaneously. So the authors design two kinds of strategies, i.e., one depends on sufficient anchors, while the other can be implemented on insufficient anchors which can be formulated as an optimal path searching problem in a graph.

Zhou et al. [8] present optimal centralized motion strategies for solving the problem of multiple mobile sensors cooperatively for tracking a moving target using distance-only measurements. The authors employ the trace of the targets position estimate covariance matrix as the object function. The performance is measured by the scope of the uncertainty for the targets position. In addition, the authors also account for the velocity constraints on mobile sensors. Different from precious work [8], the authors [9] impose constraints on the minimum distance at which the mobile sensors are allowed to be close to the target. Besides, the authors adopt measurements extend to a mixture of relative observations, including distance-only, bearing-only, and distance-and-bearing measurements.

Our previous work [10], [11] propose an integrated control approach which allows the mobile sensors adjust their positions according to sensing quality, communication quality and area coverage. In [10], Li et al. combine these three performance metrics together as the cost function. The decentralized motion algorithm in this case is based on the gradientdescent of the cost function with the constant step-size of 1 . However, the authors do not consider the speed constrains on the motion of the mobile sensors. And the work [12] extends the idea of [10]. The authors design the coordinative moving strategy which minimizes the sensing quality while guaranteeing specified coverage quality in centralized way. In addition, considering the velocity constrains on the target and the mobile sensors, the authors employ multi-step optimization and moving multiple sensors to improve the tracking performance.

In this paper, our objective is to design the coordinative moving strategy which guarantees the target can be detected in each observed step while minimizing the amount of moving sensors. Since accurately predicting the motion of the target over multiple steps is impossible, we pay much attention on the problem by which the mobile sensors are assigned the optimal destinations at each step. Specifically, in order to reduce the energy consumption throughout the network, we aim to minimize total traveled distance at each step.

The major contributions of this paper can be summarized as follows:

1) Different from previous work [6], [12], we take sensing quality, the number of moving sensors and the total traveled distance at each time step into consideration. We formulate the problem into one which aims to ensure the target will not be lost while minimizing the amount of sensors to move and then the total traveling distance of all moving sensors each step.

2) We make full use of the mobility of mobile sensors in solving the optimization tracking problem. In this framework, the tracking problem can be transformed into deployment problem and then the tracking task is formulated as assigning minimum number of mobile sensor to achieve both certain coverage requirement and the total traveled distance requirement.

3) The performance of the proposed method is evaluated by 3D display simulator. And a simulation system which is implemented for verification is close to the realistic scenarios.

\section{PROBLEM Formulation}

Assume that $M$ mobile sensors ${ }^{1}$ are deployed in FoI for cooperatively detecting the mobile targets. For simplicity, we assume that the sensors are able to detect the boundaries of FoI and stay within it.

\section{A. The Moving Model for Mobile Target}

Suppose the target moves according to the following dynamic process defined in the discrete time domain

$$
\mathbf{x}[k+1]=F \mathbf{x}[k]+\mathbf{w}[k]
$$

where $\mathbf{x}[k] \in \mathbb{R}^{n}$ is the process state in step $k$. In this paper, we consider $\mathbf{x}[k]$ is a second-order state, i.e., $\mathbf{x}[k]=$ $\left[\begin{array}{llll}x_{t}[k] & \dot{x}_{t}[k] & y_{t}[k] & \dot{y}_{t}[k]\end{array}\right]^{T}$, where $\dot{x}_{t}$ and $\dot{y}_{t}$ are the target's speed in horizontal and vertical direction respectively. Beside, $\mathbf{w}[k]$ represents the model uncertainty. In the above, $\mathbf{w}[k]$ is assumed to be zero-mean white noise with covariance $Q[k]$.

The dynamic matrix $F$, in (1) for a sampling interval of $T$ is given by

$$
F=\left[\begin{array}{llll}
1 & T & 0 & 0 \\
0 & 1 & 0 & 0 \\
0 & 0 & 1 & T \\
0 & 0 & 0 & 1
\end{array}\right]
$$

\footnotetext{
${ }^{1}$ Indexed from 1 to $M$.
} 
The covariance $Q$ is described as

$$
Q[k]=\operatorname{cov}(\mathbf{W}[k])=\left[\begin{array}{cccc}
\frac{T^{3} \phi_{s}}{3} & \frac{T^{2} \phi_{s}}{2} & 0 & 0 \\
\frac{T^{2} \phi_{s}}{2} & T \phi_{s} & 0 & 0 \\
0 & 0 & \frac{T^{3} \phi_{s}}{2} & \frac{T^{2} \phi_{s}}{2} \\
0 & 0 & \frac{T^{2} \phi_{s}}{2} & T \phi_{s}
\end{array}\right]
$$

where $\phi_{s}$ is the spectral concentration of zero-mean white noise.

\section{B. The Measurement Model}

Assume that each mobile sensor has a circular sensing range with the radius $r$. Meanwhile due to the capability of sensor, the measurement of target is modeled as

$$
\mathbf{y}[k]=\left\{\begin{array}{cl}
H \mathbf{x}[k]+\mathbf{v}_{i}[k], & d_{s t} \leq r \\
0, & \text { otherwise }
\end{array}\right.
$$

where $d_{s t}=\left\|\left(x_{t}[k], y_{t}[k]\right)-\left(x_{i}[k], y_{i}[k]\right)\right\|$ is the distance between sensor and the target, $\mathbf{y}[k] \in \mathbb{R}^{2}$ is the observation vector for the detected sensor, $\left(x_{t}[k], y_{t}[k]\right)$ stand for the target's geographical position, sensor $i$ which located at $\left(x_{i}[k], y_{i}[k]\right)$. What's more, $\mathbf{V}_{i}[k], i=1,2, \cdots, M$, is considered to be zero-mean white noise with covariance $R$. $H \in \mathbb{R}^{n \times 1}$ is the linearized measurement model matrix.

\section{Problem of Interest}

As shown in the previous section, the original problem of this paper is to design a strategy which guarantees that the target will not get lost in consecutive steps once detected while minimizing the amount of moving sensors as well as minimizing the total moving distance. Specifically, the problem can be summarized as follows:

Problem: At each step, decide the minimal number of sensors and destinations, so that the detected target would be detected at the following step with the minimum total moving distance of sensors.

The whole strategy includes four parts:

1) Estimating the current position of the target.

2) Using the target model and measurement model to predict the possible region for mobile target.

3) Determining the minimum number of mobile sensors needed and computing a set of new locations to be assigned to the mobile sensors in order to cover the whole uncertainty position.

4) Assigning each mobile sensor to one new location within the set in the way to minimize the mobile sensors' total moving distance.

\section{Problem Solution}

In this section, we propose a novel strategy to solve the original problem under consideration. The proposed method is composed of estimating and predicting the state of the mobile target, calculate the possible region for mobile target, determine the number and locations of mobile sensors, the scheme for assigning mobile sensors.

\section{A. Calculate the Possible Region for Mobile Target}

Here we assume that the target has been detected initially when it appears in the FoI as illustrated in Figure 1. With the above target model and measurement model, the detected sensor can utilize the discrete Kalman filtering to estimate and predict the target's state recursively, which consists of the following two parts:

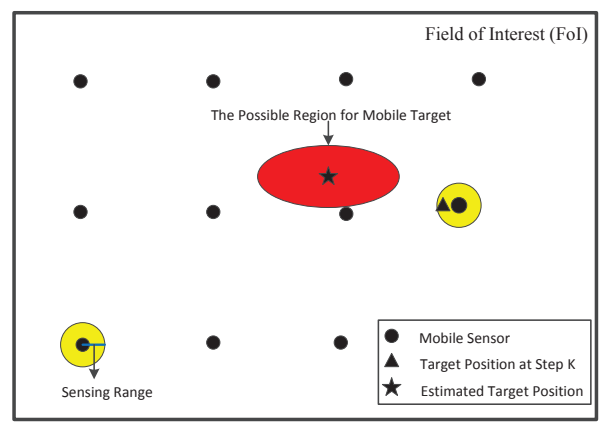

Fig. 1. An illustration of the mobile tracking: The red region is the possible region where the target will be with a high confidence.

Discrete Kalman filter time update equations (Predict):

$$
\begin{aligned}
\hat{\mathbf{x}}[k \mid k-1] & =F \hat{\mathbf{x}}[k-1 \mid k-1] \\
P[k \mid k-1] & =F P[k-1 \mid k-1] F^{T}+Q[k]
\end{aligned}
$$

Measurement update equations (Correct):

$$
\begin{aligned}
K[k] & =P[k \mid k-1] H^{T}\left(H P[k \mid k-1] H^{T}+R\right)^{-1} \\
P[k \mid k] & =(I-K[k] H) P[k \mid k-1] \\
\hat{\mathbf{x}}[k \mid k] & =\hat{\mathbf{x}}[k \mid k-1]+K[k](\mathbf{y}[k]-H \hat{\mathbf{x}}[k \mid k-1])
\end{aligned}
$$

Note that $\hat{\mathbf{x}}[k \mid k-1]$ is the predicted target state from the sensor view at step $k-1$, while $\hat{\mathbf{x}}[k \mid k]$ is the estimated state. $K[k]$ is the Kalman filter gain, $P[k \mid k-1]$ and $P[k \mid k]$ represent the corresponding prediction and estimation error covariance matrices, which are formulated as follows

$$
P=\left[\begin{array}{llll}
P_{x x} & P_{x \dot{x}} & P_{x y} & P_{x \dot{y}} \\
P_{\dot{x} x} & P_{\dot{x} \dot{x}} & P_{\dot{x} y} & P_{\dot{x} \dot{y}} \\
P_{y x} & P_{y \dot{x}} & P_{y y} & P_{y \dot{y}} \\
P_{\dot{y} x} & P_{\dot{y} \dot{x}} & P_{\dot{y} y} & P_{\dot{y} \dot{y}}
\end{array}\right]
$$

Typically, the distance measurements have a profound effect on estimation accuracy. In order to guarantee the target can be detected in each step, it is important to determine the uncertainty for the estimated position of the target. According to [8], Zhou et al. consider that target's uncertainty position is depicted by the $3 \sigma$ ellipse. It is proved that if the sensor keep still and measures the distance to the target, the sensor believes that the target is within the uncertainty region with high probability.

Once the estimates of the target have been obtained, the 
parameters of an error ellipse can be written by

$$
\begin{aligned}
& E=\sqrt{\frac{P_{x x}+P_{y y}+\left(\sqrt{\left(P_{x x}-P_{y y}\right)^{2}+4 P_{x y}^{2}}\right)}{2}} \\
& F=\sqrt{\frac{P_{x x}+P_{y y}-\left(\sqrt{\left(P_{x x}-P_{y y}\right)^{2}+4 P_{x y}^{2}}\right)}{2}}
\end{aligned}
$$

where $E$ and $F$ stand for the elliptic long half shaft and short half shaft, from which can get the error ellipse. We thus see that the region of error ellipse is related to the dynamic model of the target.

\section{B. Determine the Number and Locations of Mobile Sensors}

In this section, we present an algorithm that can determine the number and destination locations of the mobile sensors which are required to cover the region of error ellipse. The key idea is to minimize the number of mobile sensors to cover the uncertainty area of the target's position, and determine the new locations of the mobile sensors. Ming et al. in [13] has proposed three algorithms that improve sensor network performance by deploy minimum number of sensor to cover a hole, i.e., the Integer Linear Programming algorithm, the Geometry algorithm, and the Spiral-In algorithm. According to [14], [15], [16], when the communication range $r_{c}$ is at least twice the sensing range $r_{s}$ (i.e., $r_{c} \geq 2 r_{s}$ ), deploying sensors in the triangular lattice pattern is optimal, at the same time using minimum number of sensor as illustrated in Figure 2. In this paper, assuming the communication range is large enough. In order to simplify the strategy of deployment of sensors, we replace the uncertainty area of the target's position by the smallest rectangle which is able to cover the error region. As stated in above the three sensors should form an equilateral triangle with the side length $\sqrt{3} r$. According to the

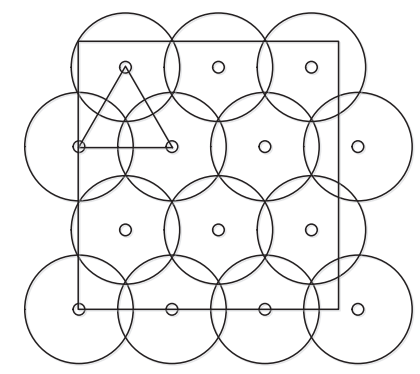

Fig. 2. Triangular lattice pattern of deployment.

geometry, we can obtain the number which is needed to cover the uncertainty area of the target's position.

The needed number of sensors in each row is given by:

$$
N_{\text {row }}=\operatorname{int}\left\lceil\frac{4 F}{3 r}+\frac{1}{3}\right\rceil
$$

The needed number of sensors in each column is given by:

$$
N_{\text {column }}=\left\{\begin{aligned}
i n t\left\lceil\frac{2 E}{\sqrt{3} r}\right\rceil, & N_{\text {row }} \bmod 2 \neq 0 \\
\operatorname{int}\left\lceil\frac{2 E+\frac{\sqrt{3} r}{2}}{\sqrt{3} r}\right\rceil, & \text { otherwise }
\end{aligned}\right.
$$

The total needed number is $N_{\text {total }}=N_{\text {row }} \times N_{\text {column }}$. Note that when $N_{\text {total }}$ is less than $M$ in each observed step, the target can be guaranteed.

\section{The Scheme for Assigning Mobile Sensors}

We regard assigning a mobile sensor as an assignment problem. The assignment problem is defined as follows: assign $N$ tasks to $N$ individuals with the least total cost, if the job $i$ is assigned to individual $j$ with a nonnegative integer cost $c_{i j}$. In our tracking system, the object of assignment problem is to assign $A$ mobile sensors to $A$ new locations with the least total moving distance. One of the most remarkable things about our assignment problem is that when the number of mobile sensors is more than the assigned new location, the $c_{i j}$ is equivalent to zero. We define the cost function as the distance between the current position of each mobile sensor and the each new location to be taken by the mobile sensors. This problem is a special case of the linear programming problem, is modeled as follows:

$$
\begin{cases}\min & z=\sum_{i, j=1}^{n} c_{i j} x_{i j} \\ \text { s.t. } & \sum_{i=1}^{n} x_{i j}=1(j=1,2, \ldots, n) \\ & \sum_{j=1}^{n} x_{i j}=1(i=1,2, \ldots, n) \\ & x_{i j}=0 \text { or } 1(i, j=1,2, \ldots, n)\end{cases}
$$

where $x_{i j}=1$ means sensor $i$ is assigned to move to location $j$, otherwise $x_{i j}=0$, sensor $i$ is not assigned to move to location $j$. The cost $c_{i j}$ form a cost matrix $C \in \mathbb{R}^{M \times M}$, which is defined as:

$$
c_{i j}=\sqrt{(r x(i)-x(j))^{2}+(r y(i)-y(j))^{2}}
$$

where $(r x(i), r y(i))$ and $(x(j), y(j))$ are respectively stand for the position of sensor $i$ and position $j$.

Recently, assignment problem has been studied extensively and many methods have been presented to solve it, such as Genetic Algorithm (GA) [17] , Ant Colony Optimization Algorithm (ACO) [18], Hungarian Algorithm, etc. GA is a heuristic searching algorithm which simulates the process of evolution and it is an effective method to find optimal and near optimal solutions for the optimization problem. ACO is able to solve combinatorial optimization problems, which produces a set of feasible solutions after each iteration. Hungarian algorithm is presented by Kuhn in 1955 [19], which focuses on find a minimum number of lines to cover all of the zeros in the reduced cost matrix. Computational complexity is a major concern in the real-time cooperative applications. Therefore, in this paper, we adopt the Hungarian algorithm to solve the mobile sensors' assignment problem.

\section{Simulations}

In order to evaluate the proposed motion strategy, we have conducted extensive simulations. In this section, we use Matlab and Samovar(which is developed by Loria, Nancy, French) [20], [21] simulator to validate our design and evaluate the performance of the proposed algorithm. 


\section{A. Matlab Simulation}

Suppose a target moving in a $100 \times 1002$ D FoI, while 16 sensors are also uniform deployed in FoI. The sensing range of sensors is set to 8 , i.e., $\forall 1 \leq i \leq 16, r_{i}=8$. The target moves simply from bottom left corner along a rectangular and then back to the initial position with varying speed, while the sampling period of sensors is $1 \mathrm{~s}$. Note that the sensor's velocity is large enough.

We have conducted 500 independent simulation trials to testify the designed strategy. Figure 3, 4 and 5 show a typical simulation result. The statistical result for the trace of error covariance is depicted in Figure 6, which is an average over 500 independent trials. It can be observed that the estimates are quite close to the true state. What's more, the trace of error covariance converge to the steady state after about 15 steps, which demonstrates the efficiency of the proposed algorithm. Furthermore, the total moving distance of mobile sensors is minimized which approaches quickly to a steady value.

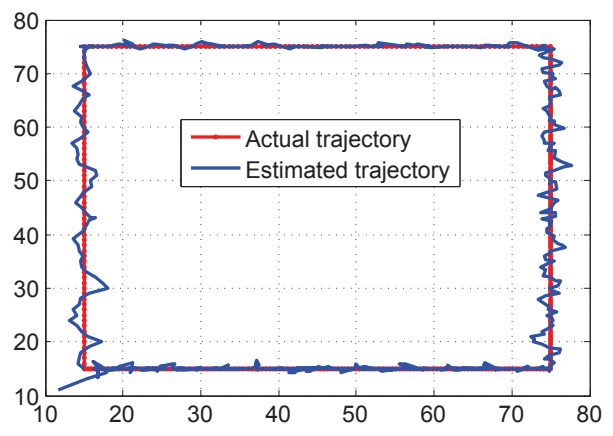

Fig. 3. The trajectory of tracking.
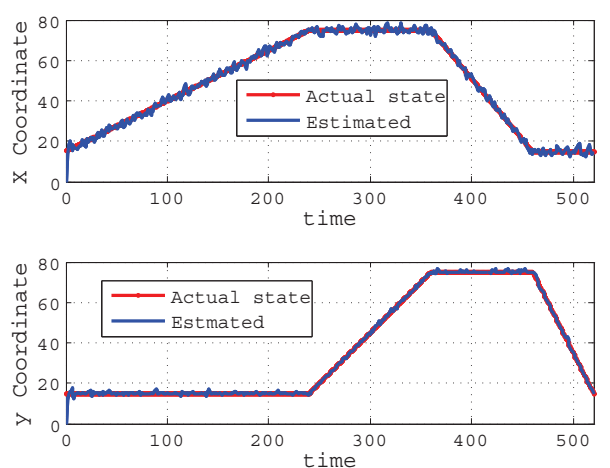

Fig. 4. The comparison of the predicted and true state of tracking. The blue line stands for the next step state

\section{B. Samovar Simulation}

Samovar is an open-source simulation platform for Wireless Sensors and Actuator Networks, which is based on Matlab/Simulink and the TrueTime library. Besides, it provides a flexible and effective tool for simulating hybrid systems with robots and wireless networks. In this section, we utilize Samovar to simulate our strategy in 3D display.

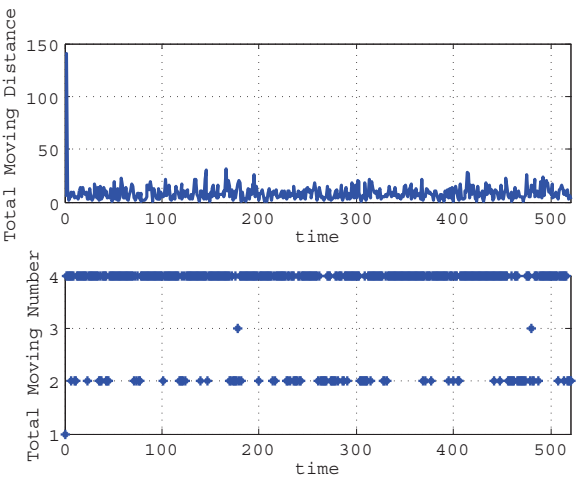

Fig. 5. Performance of total moving distance and the amount of moving sensors.

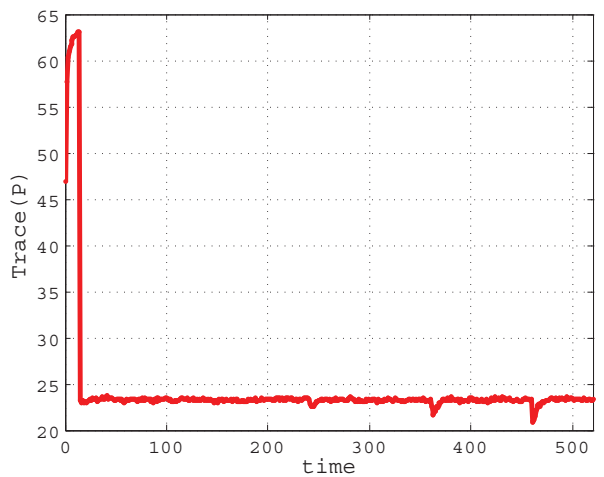

Fig. 6. The average trace of error covariance of tracking.

The setting of the simulator is as follows: a target robot moves simply from right side to left side with a constant velocity $v_{s}=0.5$, where the sensing range of sensors is set to 2. While 6 mobile robots are distributed within the FOI to track the target. The sampling period of sensors is 1s. Each mobile sensor can communicate with the base station. Each sensor periodically broadcasts a tracking message, after receiving the messages, the base station calculates the sensors positions by our algorithm. At last, the base station sends the sensors' positions through a wireless network to guide the sensors. The Simulink model is shown in Figure 7. Figure 8 shows the tracking performance at a typical step.

\section{CONCLUSION}

In this paper, we propose a coordinative scheme to guarantee the target can be detected in each observed step while minimizing the moving number of mobile sensors. The proposed strategy consists of obtaining the current position of the target and then predicting its next time-step location using secondorder dynamic model. Once the uncertainty area of the target's position is defined by the scheme of determining a uncertainty position. The mobile sensors are then allowed to cover it in an optimal way in the sense that the minimum number of sensors are used. We formulate it as the problem of deploying mobile sensor to achieve coverage requirement. The set of new sensors locations is thus defined. Each position is assigned to different 

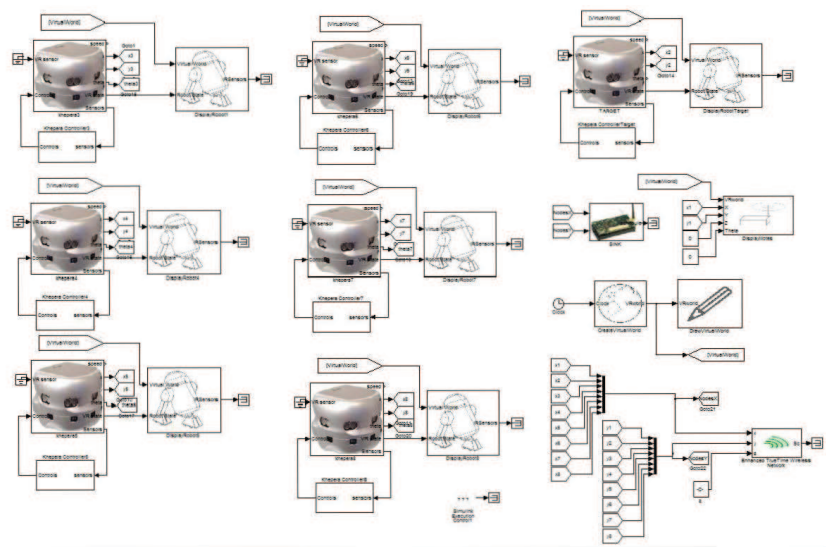

Fig. 7. Matlab/Simulink model.

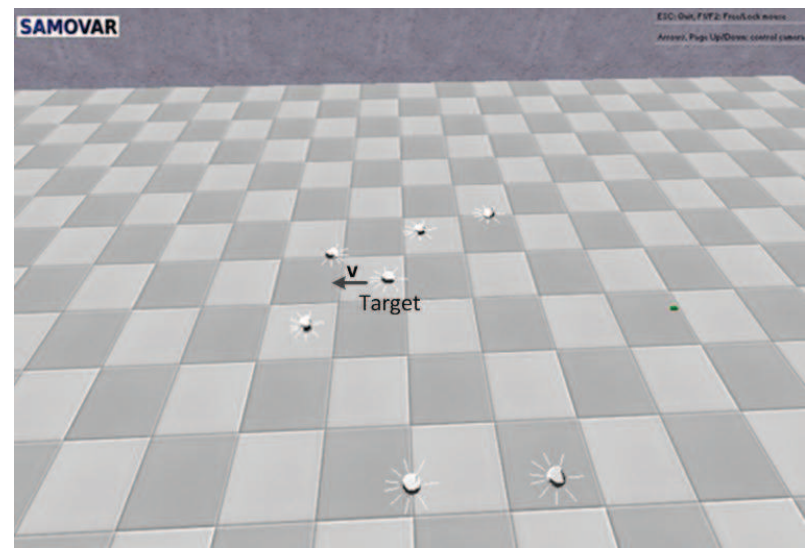

Fig. 8. Simulation result in Samovar: The results of step k.

mobile sensor so that the total traveling distance is minimized. Extensive simulations are given to evaluate performance and demonstrate the efficiency of the proposed strategy.

A straightforward extension of our work is to include more practical constraints on the motion of the target and mobile sensors. Additionally, we intend to investigate distributed scheme of the proposed method. Experimental evaluation is also our future direction.

\section{REFERENCES}

[1] Philo Juang, Hidekazu Oki, and Yong Wang. Energy-efficient computing for wildlife tracking: design tradeoffs and early experiences with zebranet. In ASPLPS'02, volume 30, pages 96-107, December 2002.

[2] Guangming Song, Yaoxin Zhou, Fei Ding, and Aiguo Song. A mobile sensor network system for monitoring of unfriendly environments. Sensors 2008, 8(11):7259-7274, 2008.

[3] Michael Winkler, Klaus-Dieter Tuchs, Kester Hughes, and Graeme Barclay. Theoretical and practical aspects of military wireless sensor networks. Journal of Telecommunications and Information Technology, 2:37-45, 2008.

[4] Mingding Han, G.N. Shirazi, Peijie Wang, and Chen Khong Tham. Mobile target tracking for healthcare applications: Trade-off between accuracy and energy. In e-health Networking, Applications and Services, 2008. HealthCom 2008. 10th International Conference, pages $206-211$, Singapore, July 2008.

[5] Yu-Chee Tseng, You-Chiun Wang, Kai-Yang Cheng, and Yao-Yu Hsieh. imouse: An integrated mobile surveillance and wireless sensor system. In Computer, volume 40, pages 60 - 66, June 2007.
[6] Farah Mourad, Hicham Chehade, Hichem Snoussi, Farouk Yalaoui, Lionel Amodeo, and Cedric Richard. Controlled mobility sensor networks for target tracking using ant colony optimization. IEEE Transactions on Mobile Computing, PP Issue:99:1-1, 2011.

[7] Pengpeng Chen, Ziguo Zhong, and Tian He. Bubble trace: Mobile target tracking under insufficient anchor coverage. In 31st International Conference on Distributed Computing Systems (ICDCS), pages 770 779, Minneapolis, MN, June 2011.

[8] Ke Zhou and Stergios I. Roumeliotis. Optimal motion strategies for range-only constrained multisensor target tracking. IEEE Transactions on Vehicular Technology, 24(5):1168-1185, 2008.

[9] Ke Zhou and Stergios I. Roumeliotis. Multirobot active target tracking with combinations of relative observations. IEEE Transactions on Vehicular Technology, 27:678 - 695, 2011.

[10] H. Li, D. Miao, J. Chen, X. Shen, and Y. Sun. Dynamic target tracking with integration of communication and coverage using mobile sensors. In Proc. Annual Conference of the IEEE Industrial Electronics Society, Porto, Portugal, pages 2636 - 2641, Nov 2009.

[11] H. Li, F. Zhang, J. Chen, and Y. Sun. Experiments on autonomous mobile sensor control for target tracking. In IEEE International Symposium on a World of Wireless Mobile and Multimedia Networks, pages $1-5$, June 2010.

[12] Peng Cheng, Xianghui Cao, Jing Bai, and Youxian Sun. On optimizing sensing quality with guaranteed coverage in autonomous mobile sensor networks. Computer Communications, 2011.

[13] Ming Zhang, Xiaojiang Du, and Kendall Nygard. Improving coverage performance in sensor networks bu using mobile sensors. In Military Communications Conference, volume 5, pages 3335 - 3341, Atlantic City, NJ, Oct 2005.

[14] Richard Kershner. The number of circles covering a set. American Journal of Mathematics, 61(3):665-671, 1939.

[15] Honghai Zhang and Jennifer C. Hou. Maintaining sensing coverage and connectivity in large sensor networks. In Ad Hoc and Sensor Wireless Networks, volume 1, pages 89-124, March 2005.

[16] Xiaole Bai, Santosh Kumar, Dong Xuan, Ziqiu Yun, and Ten H. Lai. Deploying wireless sensors to achieve both coverage and connectivity. In Proceedings of the 7th ACM international symposium on Mobile ad hoc networking and computing, pages 131-141, Florence, Italy, May 2006.

[17] P.C.Chu and J.E.Beasley. A genetic algorithm for the generalised assignment problem. Computers and Operations Research, 24(1):1723, 1997.

[18] Marco Dorigo and Luca Maria Gambardella. Ant colony system: A cooperative learning approach to the traveling salesman problem. IEEE Transactions on Evolutionary Computation, 1(1):53-66, 1997.

[19] H.W. Kuhn. The hungarian method for the assignment problem. In Naval research logistics quarterly, volume 2, pages 83-97, 1955.

[20] Samovar. Homepage. http://samovar.loria.fr/.

[21] Lionel Havet, Adrien Guenard, and Francoise Simonot-Lion. Samovar : An evaluation framework for real time applications deployment over wsans. In 15th IEEE International Conference on Emerging Technologies and Factory Automation, pages 1 - 8, Sept 2010. 